\title{
Vulvar pruritus secondary to hemochromatosis
}

\author{
Erica Hofland $\mathrm{MD},{ }^{1}$ Colleen $\mathrm{K}$ Stockdale, $\mathrm{MD}, \mathrm{MS}^{1}$
}

Keywords: Hemochromatosis, vulvar pruritus, vulvar itching

\begin{abstract}
Background: While acute pruritus is often attributable to yeast vulvovaginitis, chronic pruritus can present as the result different conditions including manifestation of a systemic disorder.
\end{abstract}

Case: A 27-year-old woman presented with a 3 year history of vulvar pruritus, burning, and dyspareunia consistent with contact dermatitis. She failed to improve despite treatment prompting evaluation for an underlying systemic disorder, such as iron deficiency anemia. Interestingly, elevated iron levels were identified leading to the diagnosis of hemochromatosis. Following treatment and near normalization of her serum iron levels, the patient's vulvar symptoms improved.

Conclusion: Vulvar pruritus is a symptom and not a disease. Thus it is important to search for the cause keeping in mind that dermatologic complaints such as pruritus can be the first manifestation of a systemic process.

${ }^{1}$ University of lowa Hospitals and Clinics, Department of Obstetrics and Gynecology, lowa City, lowa

\section{Introduction}

The approach to common causes of vulvar pruritus is based on the symptom duration (acute vs chronic), age of the patient (pre-menarche, reproductive age, or post-menopausal), and whether or not the patient has other associated symptoms. While the most common cause of acute vulvar pruritus is yeast vulvovaginitis, chronic pruritus is often the result of contact dermatitis, and/or dermatoses. It is important, however, to remain open to the possibility that vulvar pruritus may be the first manifestation of an underlying systemic disorder particularly when symptoms persist despite adequate treatment and testing remains unrevealing. ${ }^{1}$

\section{Case Report}

A 27-year-old G0 woman referred to our tertiary care center with a 3 year history of vulvar pruritus and burning incompletely relieved following application of local topical steroid creams. Additionally, she noted

Please cite this paper as: Hofland E, Stockdale CK. Vulvar pruritus secondary to hemochromatosis. Proc Obstet Gynecol. 2014;4(2): Article 9 [ 4 p.]. Available from: http://ir.uiowa.edu/pog/. Free full text article.

Corresponding author: Colleen K. Stockdale, MD, MS, University of lowa Hospitals and Clinics, Department of Obstetrics and Gynecology, 200 Hawkins Drive, lowa City, IA 52242. Telephone: 319-384-6849. Fax: 319-384-8620. colleen-stockdale@uiowa.edu.

Financial Disclosure: The authors report no conflict of interest.

Copyright: (c) 2014 Hofland \& Stockdale. This is an open-access article distributed under the terms of the Creative Commons Attribution License, which permits unrestricted use, distribution, and reproduction in any medium, provided the original author and source are credited. 
dyspareunia and recurrent posterior fourchette fissuring with attempts at vaginal penetration. Numerous contact irritants were identified including vulvar shaving, use of scented products, and prescribed creams.

Her gynecologic history was unremarkable. Menarche occurred at age 15 with regular cycles. She reported using condoms for contraception. Past medical history included irritable bowel syndrome diagnosed at age 13 years and recurrent urinary tract infections for the preceding 2-3 years. She reported consuming 2-5 alcoholic beverages weekly and denied use of tobacco or illicit substances. Her family history was unremarkable.

Her exam was remarkable for marked erythema of the labia majora extending to the vestibule circumferentially. On palpation of the pelvic floor, exquisite tenderness, elevated tone, and muscle fiber shortening of the levator ani was identified. Microscopy, $\mathrm{pH}$, and 'whiff test' of the vaginal discharge and yeast culture were performed and were all normal/negative. Her initial diagnoses included dyspareunia with contact dermatitis, and pelvic floor tension myalgia. A comprehensive treatment plan was initiated including: referral for pelvic floor physical therapy, avoidance of all contact irritants, comfort care including sitz bath, and initiation of a local steroid, triamcinolone $0.1 \%$ ointment.

When she returned for follow up in 3 months' time she reported significant improvement in pelvic floor complaints, but continued to note vulvar pruritus and burning. On exam, erythema was unchanged. However, a $2 \times 3 \mathrm{~mm}$ area of lichenification of the posterior fourchette was noted (figure 1). Repeat microscopy, $\mathrm{pH}$, and yeast cultures were again normal/negative and an excisional biopsy of the area of lichenification was performed which demonstrated findings consistent with condyloma.

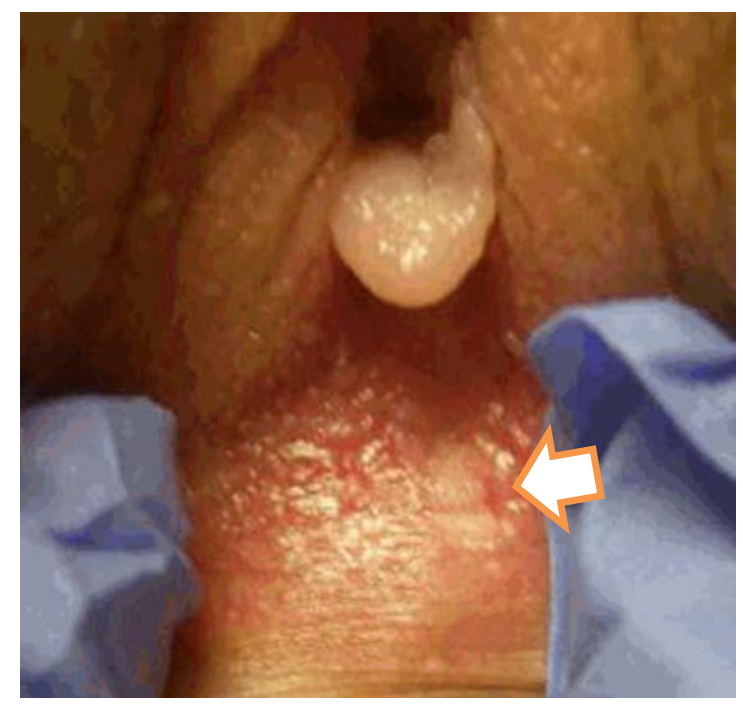

\section{Figure 1. Arrow demonstrating area of lichenification}

She continued to have waxing and waning symptoms despite avoidance of contact irritants and local steroid application. Repeat examinations continued to be negative for infectious etiology (bacterial vaginosis, trichomonas, yeast, herpes simplex). Hydroxyzine was advised for the refractory vulvar symptoms, and provided mild relief. However, about 15 months after the initial evaluation, her symptoms became so significant that she was no longer able to apply topical medications without aggravating symptoms of vulvar irritation, burning, and pruritus. Throughout this time the labia and vestibule continued to appear 
erythematous on exam and repeat evaluations including microscopy, $\mathrm{pH}$, and yeast cultures returned negative. Allergy testing was discussed with the patient as was epicutaneous patch testing to evaluate for possible underlying sources for the persistent symptoms. She was not able, however, to pursue allergy testing. Rather, evaluation was initiated for a possible underlying systemic disorder given the refractory nature of her symptoms (e.g. vitamin deficiency, iron deficiency, thyroid dysfunction). Interestingly, we identified an elevated serum iron, iron saturation, and ferritin consistent with hematochromatosis rather than deficiency as anticipated (Table 1).

Table 1. Initial laboratory studies

\begin{tabular}{|l|c|c|}
\hline Component & Value & Range \\
\hline Vitamin B12 & 398 & $243-894 \mathrm{pg} / \mathrm{mL}$ \\
\hline $\begin{array}{l}\text { Vitamin D (25 } \\
\text { hydroxy) }\end{array}$ & 31 & $20-80 \mathrm{ng} / \mathrm{mL}$ \\
\hline TSH Iron & 1.35 & $\begin{array}{c}0.27-4.20 \\
\mathrm{uIU} / \mathrm{mL}\end{array}$ \\
\hline Iron Serum & 212 & $72-130 \mathrm{mcg} / \mathrm{dL}$ \\
\hline $\begin{array}{l}\text { Total Baling Capacity } \\
\text { Binding }\end{array}$ & $\begin{array}{c}224-429 \\
\mathrm{mcg} / \mathrm{dL}\end{array}$ \\
\hline Iron Saturation & 241.2 & $\begin{array}{c}13.0-150 \\
\mathrm{ng} / \mathrm{mL}\end{array}$ \\
\hline Ferritin & & \\
\hline
\end{tabular}

Given the abnormal screening results, the patient was referred to a gastroenterologist for further evaluation. Genetic testing performed confirmed patient was homozygous for $\mathrm{C} 282 \mathrm{Y}$ mutation, the most common mutation of hemochromatosis. Treatment with serial phlebotomy was initiated shortly thereafter. Four months after initiating treatment for hematochromatosis the patient returned to our center and reported a 90\% improvement in her vulvar symptoms; with only mild residual vulvar irritation and burning and her vulvar exam was normalized (no erythema). Her ferritin was within normal range and her iron level had improved although still remained mildly elevated (Table 2). She planned to continue treatment as outlined by her local gastroenterologist for intermediate and long-term management.

\section{Table 2. Ferritin and Iron studies four} months after initiating treatment

\begin{tabular}{|l|c|c|}
\hline Component & Value & Range \\
\hline Iron Serum & 167 & $72-130 \mathrm{mcg} / \mathrm{dL}$ \\
\hline Ferritin & 148.6 & $\begin{array}{c}13.0-150.0 \\
\mathrm{ng} / \mathrm{mL}\end{array}$ \\
\hline
\end{tabular}

\section{Discussion}

The temporal marked improvement of vulvar symptoms associated with normalization of serum ferritin following initiation of treatment for hematochromatosis in this patient supports elevated iron as a likely causative factor for this patient's localized vulvar symptoms. Although condyloma was noted on the excisional biopsy, the symptoms worsened over the subsequent 15 months while the exam remained unremarkable except for erythema. Thus, human papilloma virus infection was not felt to be likely associated with her persistent symptoms.

While vulvar pruritus and irritation is a common and distressing condition that affects most women at some point in their lives, pruritus as a presentation of 
hematochromatosis is rare. We identified 3 case reports with generalized pruritus as the major symptom prior to diagnosis of hematochromatosis on a Pub Med search. ${ }^{2-4}$ To our knowledge this is the only report where localized vulvar pruritus has been the presenting complaint prior to the evaluation and diagnosis of hemochromatosis. Rather, we were anticipating iron deficiency anemia as a possible systemic etiology for her persistent pruritus. ${ }^{1}$ As noted with the prior case reports, the temporal improvement in symptoms after near normalization of her serum iron and ferritin levels suggest that the pruritus was primarily due to the underlying hematochromatosis. ${ }^{3,4}$ Thus, these cases demonstrate how extremes of serum iron levels may correspond with vulvar itching and irritation.

\section{Conclusion}

Vulvar pruritus is a symptom and not a disease. Thus it is important to search for the cause keeping in mind that dermatologic complaints such as pruritus can sometimes be the first presentation of a systemic disease process.

Acknowledgement: This case was presented at the ACOG 2013 Annual Clinical Meeting "Stump the Professors" by Dr. Hofland.

\section{References}

1. Bohl TG. Overview of vulvar pruritus through the life cycle. Clin Obstet Gynecol. 2005 Dec;48(4):786-807. PubMed PMID: 16286826.

2. Nestler JE. Hemochromatosis and pruritus. Ann Intern Med. 1983 Jun;98(6):1026. http://dx.doi.org/10.7326/0003-4819-986-1026_1. PubMed PMID: 6859694.

3. Hamilton DV, Gould DJ. Generalized pruritus as a presentation of idiopathic haemochromatosis. Br J Dermatol. 1985 May;112(5):629. http://dx.doi.org/10.1111/j.13652133.1985.tb15277.x. PubMed PMID: 4005161.

4. Kluger $\mathrm{N}$, Raison-Peyron $\mathrm{N}$, Rigole $\mathrm{H}$, Bessis D, Blanc F, Guillot B. Generalized pruritus revealing hereditary haemochromatosis. Acta Derm Venereol. 2007;87(3):277. PubMed PMID: 17533502. 